\title{
Discretization and Iterative Solution Techniques for Elliptic Problems on Non- Matching Grids
}

R. Lazarov and J. Pasciak

November 7, 2000

U.S. Department of Energy

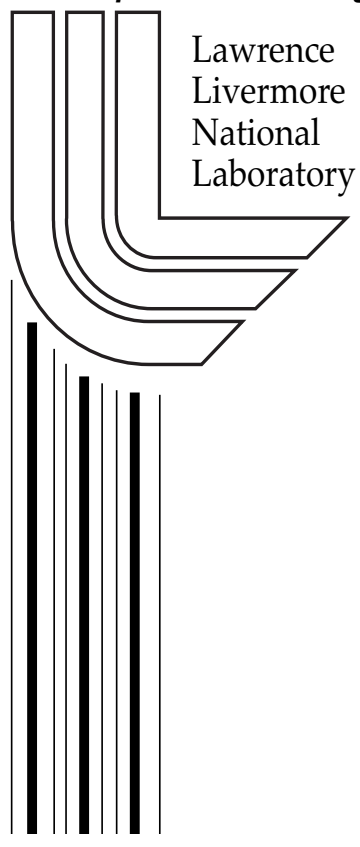




\section{DISCLAIMER}

This document was prepared as an account of work sponsored by an agency of the United States Government. Neither the United States Government nor the University of California nor any of their employees, makes any warranty, express or implied, or assumes any legal liability or responsibility for the accuracy, completeness, or usefulness of any information, apparatus, product, or process disclosed, or represents that its use would not infringe privately owned rights. Reference herein to any specific commercial product, process, or service by trade name, trademark, manufacturer, or otherwise, does not necessarily constitute or imply its endorsement, recommendation, or favoring by the United States Government or the University of California. The views and opinions of authors expressed herein do not necessarily state or reflect those of the United States Government or the University of California, and shall not be used for advertising or product endorsement purposes.

Work performed under the auspices of the U. S. Department of Energy by the University of California Lawrence Livermore National Laboratory under Contract W-7405-Eng-48. 


\title{
FINAL REPORT ON THE CONTRACT
}

\section{DISCRETIZATION AND ITERATIVE SOLUTION TECHNIQUES FOR ELLIPTIC PROBLEMS ON NON-MATCHING GRIDS}

\author{
PRINCIPAL INVESTIGATORS: RAYTCHO LAZAROV AND JOSEPH PASCJAK
}

FUNDED BY LLNL FOR THE PERIOD JUNE 1, 2000 .OCTOBER 30, 2000

\begin{abstract}
Construction, analysis and numerical testing of efficient solution techniques for solving elliptic PDEs that allow for parallel implementation have been the focus of the research. A number of discretization and solution methods for solving second order elliptic problems that include mortar and penalty approximations and domain decomposition methods for finite elements and finite volumes have been investigated and analyzed. Techniques for parallel domain decomposition algorithms in the framework of PETC and HYPRE have been studied and tested. Hierarchical parallel grid refinement and adaptive solution methods have been implemented and tested on various model problems.
\end{abstract}

\section{INTRODUCTION}

The discretization methods of PDEs on non-matching grids have become an important direction for research in the applied scientific computing. This approach provides greater flexibility in the grid generation process, increases the portability of various approximation methods and computer implementations, enhances the capabilities of coarsening strategy in parallel algebraic multigrid methods, and provides a natural and practical way for parallel domain decomposition methods and parallel adaptive methods based on a posteriori error analysis. Our research focussed on the analysis and testing of some methods from this promising approach in order to make competitive implementations in engineering codes for complex applications.

\section{MORTAR APPROXIMATIONS OF FINITE ELEMENT AND FINITE VOLUME METHODS}

\section{ON NON-MATCHING GRIDS}

We have completed a comprehensive study and testing of various mortar spaces which can be used in non-conforming domain decomposition methods. This in general means that the meshes from the subdomains do not necessarily match along the interface and the continuity of the solution along the subdomain interfaces is imposed in a weak sense. The mortar space provides the "glue" for this process. The mortars have a meaning of trace of the co-normal derivative of the solution at the subdomain interface

1991 Mathematics Subject Classification. 65F10, 65N20, 65N30.

Key words and phrases. discretization methods, non-matching grids, multilevel methods, iterative methods, domain decomposition. 
understood in a sense of functions in space $H_{-}{ }^{1 / 2}$, so a reasonable mortar spaces may contain discontintious functions. However, most of the finite element approximations of the mortar space used in the mortar finite element method have been related to the traces of the finite element spaces on the interfaces, which result in continuous function. We have constructed mortar spaces by using the dual bases approach and some finite volume ideas. Such constructions are relatively simple, valid for general meshes and lead to local computations. We have constructed discontinuous local dual basis functions for the mortar space which lead to diagonal mass matrices and hence to simple computational schemes. Our mortar spaces, presented and justified in [2] have been tested on a series of 3-D problems that include second order elliptic equations and problems of linear elasticity.

\section{DOMAIN DECOMPOSITION METHODS FOR NON-MORTAR APPROXIMATIONS ON}

\section{NON-MATCHING GRIDS}

The interior penalty method aims at eliminating the need for additional (Lagrange multiplier or mortar) spaces and imposes (only approximately) the required continuity across the interfaces by appropriate penalty term (completed in [5]). In our approach the jumps in the values of the functions along these interfaces is penalized in the variational formulation. For smooth solutions we lose the optimal accuracy due to lower approximation at the interface, but on the other hand we produce symmetric and positive definite discrete problem which has optimal condition number.

We also address the issue of constructing preconditioners for solving the system on the composite non-matching grids. We proposed and investigated an interface domain decomposition type preconditioner, that is spectrally equivalent to the reduced on the interface algebraic problem. We have tested both the accuracy and the preconditioned method on a series of model problems.

Similar procedure for combining mixed amid Galerkin problems does not require a mortar space. Methods for solving the corresponding composite problems have been proposed, studied and tested in [6].

\section{MULTILEVEL ADAPTIVE GRID REFINEMENT AND ERROR CONTROL}

We worked on a problem of multilevel grid refinement and error control for both finite volume approximations and penalty domain decomposition methods. This work is in direct connection with research in CASC on developing and testing of parallel algorithms (J. Jones, V. Henson. R. Falgout, U. Meier, C. Tong, and P. Vassilevski). We have developed 2- and 3-D codes for parallel adaptive grid refinement that produces nested (and matching) grids. The obtained multi-level structure is used to define multigrid preconditioners. In a closed collaboration with Dr. Panayot Vassilevski and Dr. Charlcs Tong the developed software was connected to HYPRE Preconditioncr Library. The connection is implemented using The Finite-Element Interf ace (EEl) specification, which provides a layered abstraction that minimizes the concern for the internal details in the HYPRE library. The connection is implemented using the Finite Element Interf ace (EEl) specification, which provides a 
layered abstraction that minimizes the concern of the internal details in the HYPRE library. The initialization

is done in parallel. GUI, using Motif, has been developed to utilize the selection of different HYPRE options. After the solution is obtained on certain level it is sent directly through AF_INET socket to visualizer (SG, developed by Dr. Michael Holst) residing on the users machine. This idea is very efficient for real time visualization of time dependent problems.

Time developed software was used for testing various ideas and strategies in the a posteriori error analysis and error control for convection-diffusion-reaction problems in 3-D domains with complex structure (see, e.g. [4]).

\section{INTERACTION WITH CASC MEMBERS, VISITORS, AND SUMMER STUDENTS}

Raytcho Lazarov and Joseph Pasciak visited CASC for the month of July. 2000. They were also in a close contact with J. Jones, V. Henson, R. Falgout, U. Meier. C. Tong, and P. Vassilevski and interacted with other long and short term visitors R. Bank, M. Holst, T. Manteuffel, S. McCormick, and G. Douglas, as well as with the summer students C. Kim, A. Iontcheva, S. Tomov, and M. Flanagan.

Three $\mathrm{Pb}$. D. students from the program of computational mathematics at Texas A\&M University, M. Flanagan, C. Kim and S. Tomov, spent two months at CASC LLNL on a professional summer internship and training. Three TAMU Ph. D. students. C. Kim. T. Kolev. and S. Tomov participated directly in the work of the contract.

\section{FURTHER COLLABORATION BETWEEN TAMU AND CASC}

Time Numerical Analysis group at TAMU has expanded and deepened the collaboration with CASC LLNL. The project integrated and enhanced the efforts of TAMU and CASC groups in construction, analysis and numerical testing of efficient, including parallel, discretization techniques and adaptive grid refinement for solving elliptic PDEs in 3-D. Our further goals are to explore and test domain decomposition type of algorithms and their parallel implementation on multiprocessor computer architectures.

The developed software for adaptive parallel grid generation that is connected to HYPRE Preconditioner Library will be used as an environment for large scale computations by the whole Numerical Analysis Group at Texas A\&M University, which includes several faculty members and more than $10 \mathrm{Ph}$. D. students.

We shall focus in the future on developing multigrid-like (MG, AMG, multilevel) algoritiin-ms that exploit coarse finite element spaces (or coarse grids) that do riot necessarily match across subdomain boundaries. In the first, more traditional one. which is based on geometric finite element discretization, the refinement, local and adaptive, will be the main direction. In order to allow for efficient parallelization, we will investigate approaches of independent refinement in each subdomain thus facing time problem of non-matching grids across the subdomain interfaces. In the dual approach, which is related to AMGe (algebraic multigrid method for finite element problems) one needs to create coarse spaces (or coarse grids) iii parallel, and one possibility is to perform any available sequential coarsening in each subdomain and thus creating non-matching coarse grids across the subdomain boundaries. That is, in both approaches one has to resolve the issue of matching the independently created problems at the intermediate grids, which will be time main objective in the 
proposed research.

As a result we plan to develop efficient parallelizable, scalable algorithms for treating both the geometrically refining spaces or algebraically coarsening grids. In the geometric approach, the error analysis as well as the convergence properties of the underlined domain decomposition type MG algorithm will be studied in details. The future research should offer alternatives to the existing efforts in CASC for parallel AMG(e) method, which might be more efficient since it exploits the additional properties of the matrices that arise in discretization procedure.

\section{REFERENCES}

[1] R.E.Bank and M. Holst, A new paradigm for parallel adaptive meshing algorithm, University of California San Diego, Preprint 1998. ftp://sdna3.ucsd.edu/pub/reb/reports/a85.ps.gz.

[2] C. Kim, R.D. Lazarov, J.E. Pasciak, and P.S. Vassilevski, Multiplier spaces for the mortar finite element method is three dimensions, (submitted to SIAM J. Numer. Anal.).

[3] RD. Lazarov, J.E. Pasciak, and S.Z. Tomov, Error control, local grid refinement, and efficient solution algorithms for singularly perturbed problems, Analytical and Numerical Methods for Singularly Perturbed Problems, Nuova Science Publishing House, 2000.

[4] RD. Lazarov and S.Z. Tomov, Adaptive finite volume element method for convection-diffusion-reaction problems in 3-D, Technical Report ISC-00-02-MATH, Texas A\& M University (to appear in Proc. Second Workshop on Large Scale Scientific Computing. Canada, June 2000).

[5] RD. Lazarov, S.Z. Tomov, and P.S. Vassilevski Interior penalty discontinuous approximations of elliptic problems, Technical Report ISC-00-04-MATH, Texas A\& M University (submitted to SIAM J. Sd. Comp.)

[6] R.D. Lazarov, J.E. Pasciak, and P.S. Vassilevski, Iterative solution of combined mixed and standard Galerkin discretizations for elliptic problems, (to appear in Numer. Linear Alg .Appl.).

DEPARTMENT OF MATHEMATICS, TEXAS A \& M UNIVERSITY, COLLEGE STATION, TX 77843.

U.S.A.

E-mail address: lazarov@math.tamu.edu,pasciak@math.tamu.edu 